An nth-order, Gaussian Energy Distribution Model for Sintering

A. K. Burnham

September 2, 2004

Chemical Engineering Journal 
This document was prepared as an account of work sponsored by an agency of the United States Government. Neither the United States Government nor the University of California nor any of their employees, makes any warranty, express or implied, or assumes any legal liability or responsibility for the accuracy, completeness, or usefulness of any information, apparatus, product, or process disclosed, or represents that its use would not infringe privately owned rights. Reference herein to any specific commercial product, process, or service by trade name, trademark, manufacturer, or otherwise, does not necessarily constitute or imply its endorsement, recommendation, or favoring by the United States Government or the University of California. The views and opinions of authors expressed herein do not necessarily state or reflect those of the United States Government or the University of California, and shall not be used for advertising or product endorsement purposes. 


\title{
An nth-order, Gaussian Energy Distribution Model for Sintering
}

\author{
Alan K. Burnham* \\ Lawrence Livermore National Laboratory \\ P. O. Box 808, L-282 \\ Livermore, CA 94551
}

\begin{abstract}
Although it is well known that the rate of sintering is governed by deceleratory kinetics, it is often difficult to fit power-law and nth-order reaction models over broad timetemperature ranges. This work shows that a phenomenological model combining a reaction order with an activation energy distribution can correlate surface area as a function of sintering time and temperature over a greater range of those variables. Qualitatively, the activation energy distribution accounts the dependence of free energy on particle size and material defects, while the reaction order accounts for geometric factors such as a distribution of diffusion lengths. The model is demonstrated for sintering of hydroxyapatite using data of Bailliez and Nzihou (Chem. Eng. J. 98 (2004), 141-152).
\end{abstract}

Keywords: sintering, kinetics, activation energy distributions, modeling, hydroxyapatite

\section{Introduction}

Sintering of powders is an industrial practice spanning many applications, and German $^{1}$ gives an excellent introduction. In very general terms, sintering is governed by two interrelated properties, a change in free energy, which provides the driving force for the process, and kinetics, which provides the mobility of the system to the lower free energy state. Sintering can occur by many mechanisms, including viscous flow, plastic flow, evaporation-condensation, surface diffusion, volume diffusion, and grain-boundary diffusion.

Predicting the kinetics of sintering has practical aspects for both process optimization and material lifetime prediction. Process optimization is usually easier, because it is usually an interpolation problem for which the calibration data might cover a relatively narrow range of conditions. Lifetime prediction is more challenging, since it involves extrapolation of artificial aging experiments outside the range of calibration. Consequently, a relatively small deviation in a model at the extremes of the calibration data can result in a relatively large error in lifetime prediction if the functional form is not correct.

A vast literature exists on the kinetics of sintering, and various equations have been derived that use powers of time and particle size along with an Arrhenius temperature dependence. ${ }^{1}$ However, these models often have difficult correlating sintering data over wide ranges of time and temperature. For example, the common $n$ thorder sintering model often requires $n$ to be a function of temperature, with the qualitative

*Corresponding author. tel. 915-422-7304; fax 925-424-3281.

E-mail address: burnham1@llnl.gov 
justification that the mechanism is changing as a function of temperature (e.g., Bailliez and Nzihou').

Changes in free energy driving the sintering process are ordinarily attributed to changes in the radius of curvature. Less widely recognized is that sintering often starts with very imperfect crystals that have free energies substantially different from the perfect material. For example, Rogers and Dinegar ${ }^{3}$ report that heats of fusion of pentaerythritol tetranitrate (PETN) crystals can have heats of fusion up to $20 \%$ less than the single crystal value, and the variation of the heat with crystallization conditions is far greater than changes in surface area caused by grinding. A free energy distribution in the starting material will result in an activation energy distribution in the kinetics.

Both $n$ th-order and activation energy distribution kinetic models have been used extensively for modeling fossil fuel conversions. ${ }^{4}$ The earliest and simplest energy distribution model used a Gaussian distribution characterized by a mean, $E_{0}$, and standard devaiation, $\sigma^{5}$ For systems with modest distributions of reactivity, $n$ th-order $(n \leq 2)$ or Gaussian ( $\sigma \leq 3 \%$ of $E_{0}$ ) models work equally well, even with considerable extrapolation in temperature. However, sintering is often, if not usually, characterized by reaction orders that are considerably larger, and possibly by free energy distributions as well, if the Rogers-Dinegar result ${ }^{3}$ for PETN is typical.

The hypothesis tested in this paper is that the temperature dependence of the reaction order can be removed if the kinetic model also includes an activation energy distribution. We use the data of Bailliez and Nzihou, ${ }^{2}$ since it covers such a broad range of temperature and degree of sintering. We find that the $n$ th-order/Gaussian distribution model works very well, resulting in a several-fold reduction in nonlinear-regression residuals compared to using either single-n or Gaussian distribution models alone.

\section{Sintering Models}

Deceleratory sintering reactions are often characterized by a power law in time $e^{6,7}$

$$
\alpha \propto(1+a t)^{-v}
$$

where $\alpha$ is the extent of reaction (e.g., ratio of the change in surface area to the ultimate change in surface area, or $1-S / S_{0}$ ) and $a$ and $v$ are constants, and $t$ is time; or an nth-order reaction, ${ }^{2,8,9}$

$$
-\mathrm{d} \alpha / \mathrm{d} t \propto(1-\alpha)^{n}
$$

Coming from different fields, Raynaud et al. ${ }^{10}$ and Tarutis ${ }^{11}$ independently note that the two approaches are actually equivalent, with the exponent of the power law in time being related to the order of the $n$ th-order reaction by $n=1+1 / v$. Reaction order is commonly interpreted in geometric terms, e.g., shrinking-core reactions are described by $n<1 .{ }^{12}$ Tarutis, drawing upon earlier work by Boudreau and Ruddick, ${ }^{13}$ notes that an $n$ th-order reaction is mathematically equivalent to for $n>1$ to a Gamma (near-exponential) distribution of reactivity. Consequently, one can consider reaction order as a measure of a distribution of diffusion lengths, for example. Regardless of the precise physical interpretation, $n$ should be constant if the geometric progression of the reaction is 
independent of temperature, and all temperature dependence would be ascribed to a single activation energy, if one uses the standard Arrhenius rate law.

Alternatively, one can use a Gaussian distribution of activation energies to describe the distribution of reactivity. In this case,

$$
\mathrm{d} \alpha / \mathrm{dt}=(1-\alpha) \int_{0}^{\infty} k(E) \exp \left[-\int_{0}^{\mathrm{t}} k(E) \mathrm{d} t\right] D(E) \mathrm{d} E
$$

where $k=A \exp (-E / \mathrm{R} T), E$ is the activation energy, $A$ is the frequency factor, $\mathrm{R}$ is the gas constant, and

$$
D(E)=(2 \pi)^{-1 / 2} \sigma^{-1} \exp \left[-\left(E-E_{0}\right)^{2} / 2 \sigma^{2}\right]
$$

where $E_{0}$ is the mean energy and $\sigma$ is the standard deviation. Eq. (3) is actually simpler than it first appears. It is implemented by discretizing the distribution into 11 to 21 parallel independent nth-order reactions, depending on the magnitude of $\sigma$, having evenly spaced energies and weighting factors calculated from a Gaussian distribution.

Both approaches yield deceleratory curves at constant temperature, with the ultimate extent of reaction appearing to depend on temperature when $n$ and $\sigma$ are large. Calculations for intermediate values of both $n$ and $\sigma$ are given in Figure 1. In order to have the overall degree of sintering cover the same range, a higher mean energy is needed for the Gaussian model so that the lowest energy channel of the distribution is close to the single value of the nth-order reaction. The shape of the deceleration and how it varies with temperature is different for the two models.

\section{Sintering of hydroxyapatite}

Bailliez and Nzihou ${ }^{2}$ provide an interesting data set for testing the ability of a combined nth-order activation energy distribution model to correlate the extent of sintering over a very wide range. They present data for two hydroxyapatites: $\mathrm{HAP}_{\mathrm{TCP}}$ was formed by reacting $\mathrm{CaCl}_{2}, \mathrm{H}_{3} \mathrm{PO}_{4}$, and $\mathrm{NaOH}$; and $\mathrm{HAP}_{\mathrm{CaO}}$ was formed by reacting $\mathrm{Ca}\left(\mathrm{NO}_{3}\right)_{2}$ with $\left(\mathrm{NH}_{4}\right)_{2} \mathrm{HPO}_{4}$ and ammonia. The initial surface areas were 28 and 104 $\mathrm{m}^{2} / \mathrm{g}$, respectively.

Data was digitized from the published plots of surface area versus time at various temperatures. It was then fitted by nonlinear regression to nth-order, Gaussian, and combined models using the LLNL analysis program Kinetics $05 .{ }^{4}$ Results of this analysis are given in Table 1. A graphical comparison of data with calculation is given in Figure 2 for the combined model.

When only one of the two parameters ( $n$ or $\sigma$ ) is used, a better fit is obtained with the $n$ th-order model for $\mathrm{HAP}_{\mathrm{TCP}}$ and with the Gaussian model for $\mathrm{HAP}_{\mathrm{CaO}}$. However, the best fit is obtained for both materials using both model parameters, and it is especially better for $\mathrm{HAP}_{\text {TCP. }}$ The mean activation energy for the Gaussian model is higher than for the nth-order model as explained in the previous section.

The activation energies reported here are about one-third lower than those

reported by Bailliez and Nzihou ${ }^{2}$. The reason for this discrepancy is not certain, but it probably relates to the common problem of deriving activation energies under 
circumstances where the extent of conversion is not maintained constant. In this case, the activation energy can shift from its true value to make up for other model deficiencies. ${ }^{14}$ This is not possible by our method, since the entire data set is fitted simultaneously to the model.

\section{Conclusions}

The combination of an $n$ th-order reaction model with a Gaussian activation energy distribution provides a simple yet powerful method for correlating sintering data over a very wide time-temperature range. The $n$ th-order aspect can be interpreted in terms of standard neck-growth phenomena and a distribution of diffusion lengths. The activation energy distribution reflects the distribution of free energies for imperfect starting crystals. While the combined model fits the data better than either aspect independently for both hydroxyapatite samples examined, the activation energy distribution aspect is considerably more important for the higher surface area material. The activation energies derived by nonlinear regression to the entire range of conversion simultaneously are more reliable than other forms of model fitting that sample different aspects of the reaction at different temperatures.

\section{Acknowledgement}

This work was performed under the auspices of the USDOE by the UC Lawrence Livermore National Laboratory under contract number W-7405-Eng-48.

Table 1. Hydroxyapatite kinetic parameters derived by nonlinear regression for the Gaussian and $n$ th-order reaction models from the data of Bailliez and Nzihou ${ }^{2}$.

\begin{tabular}{|l|c|c|c|c|c|}
\hline & $A, \mathrm{~s}^{-1}$ & $E_{0} / R, \mathrm{~K}$ & $n$ & $\sigma, \%$ of $E_{0}$ & $\mathrm{RSS}^{\mathrm{a}}$ \\
\hline \multicolumn{1}{|c|}{$\mathrm{HAP}_{\mathrm{TCP}}$} & & & & & \\
\hline$n$ th-order & $8.43 \times 10^{11}$ & 30313 & 6.89 & 0.0 & 0.1001 \\
\hline Gaussian & $1.63 \times 10^{11}$ & 32508 & 1.00 & 17.2 & 0.3090 \\
\hline Both & $1.64 \times 10^{13}$ & 33001 & 7.01 & 6.93 & 0.0762 \\
\hline \multicolumn{1}{|c|}{$\mathrm{HAP}_{\mathrm{CaO}}$} & & & & & \\
\hline$n$ th-order & $1.79 \times 10^{9}$ & 24246 & 4.01 & 0.0 & 0.1530 \\
\hline Gaussian & $9.44 \times 10^{9}$ & 27383 & 1.00 & 11.4 & 0.1189 \\
\hline Both & $2.92 \times 10^{10}$ & 27149 & 3.22 & 8.32 & 0.1046 \\
\hline
\end{tabular}

${ }^{\mathrm{a}}$ Residual sum of squares from nonlinear regression 


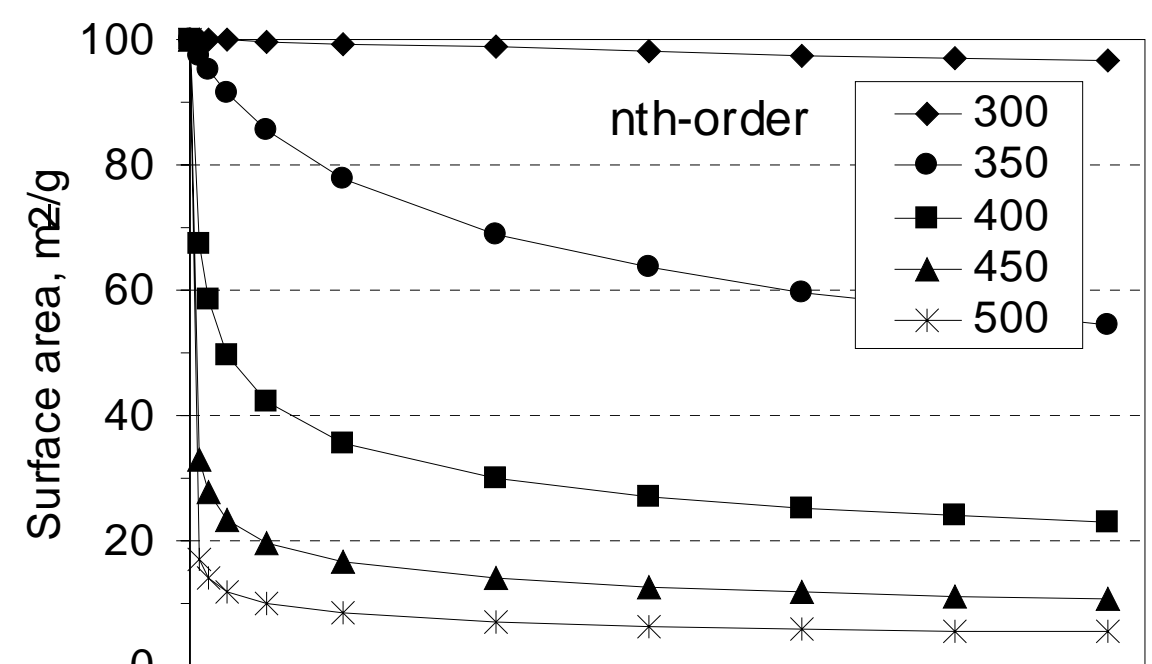

0

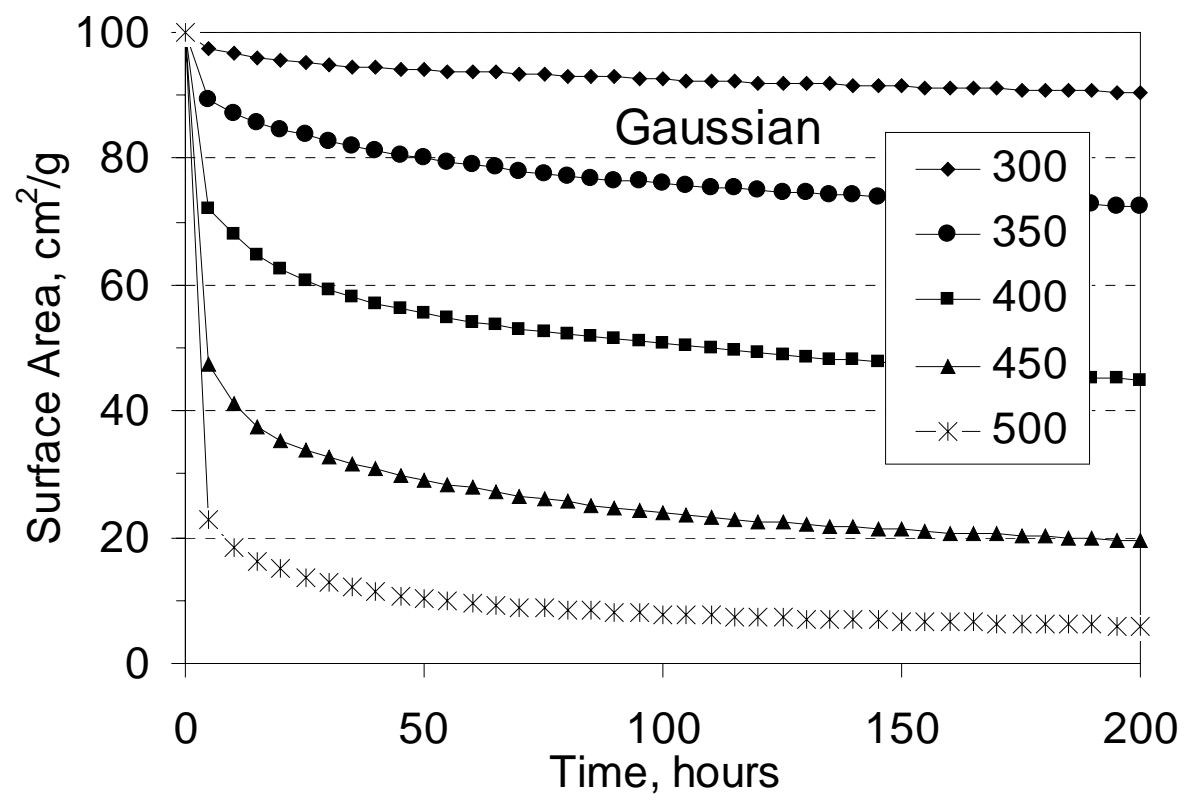

Fig. 1. Idealized sintering curves calculated from nth-order (top) and Gaussian activation energy distribution (bottom) models. In both cases, $A=3 \times 10^{15} \mathrm{~s}^{-1}$. For the $n$ th-order model, $n=5$ and $E / R=30,000 \mathrm{~K}$. For the Gaussian model, $\sigma=10 \%$ of $E_{0}$ and $E_{0}=$ $33,000 \mathrm{~K}$. The higher mean energy is needed for the Gaussian model so that the lowest energy channel of the distribution is close to $30,000 \mathrm{~K}$. 


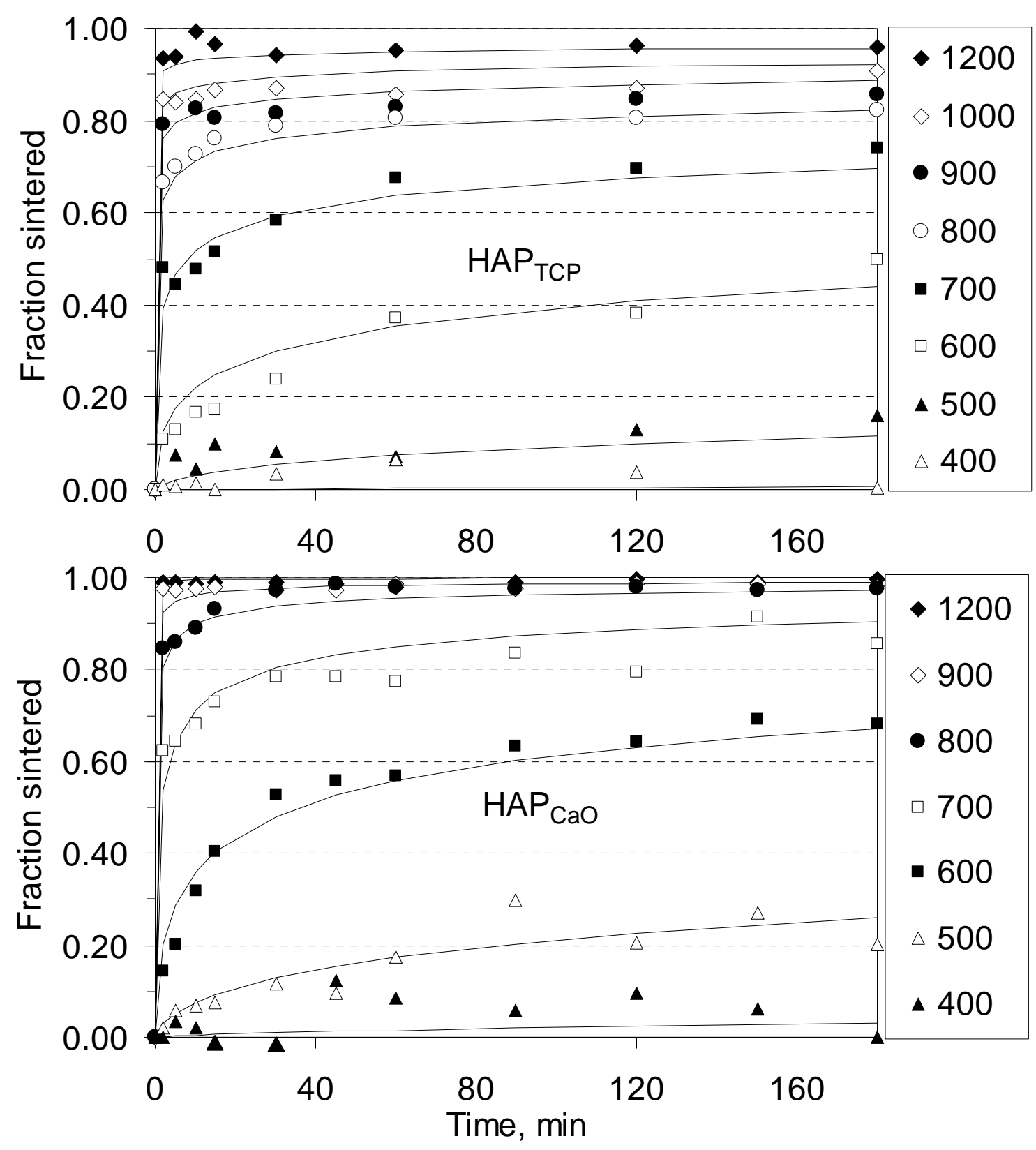

Figure 2. Comparison of measured and calculated fractions sintered $\left(1-S / S_{0}\right)$ for the nthorder Gaussian energy distribution model. The four model parameters are fitted simultaneously by nonlinear regression and are given in Table 1. 


\section{References}

${ }^{1}$ R. M. German, Sintering theory and practice, John Wiley \& Sons, 1996.

${ }^{2}$ S. Bailliez, A. Nzihou, Chem. Eng. J. 98 (2004) 141.

${ }^{3}$ R. N. Rogers, R. H. Dinegar, Thermochim. Acta 3 (1972) 367.

${ }^{4}$ A. K. Burnham, R. L. Braun, Energy Fuels 13 (1999) 1.

${ }^{5}$ D. B. Anthony, J. B. Howard, AICHE J. 22 (1976) 625.

${ }^{6}$ W. D. Kingery, H. K. Bowen, D. R. Ulmann, Introduction to Ceramics, Chapter 10, John Wiley \& Sons (1976).

${ }^{7}$ R. M. German, J. Am. Cer. Soc. 61 (1978) 272.

${ }^{8}$ W. G. Schlaffer, C. Z. Morgan, J. N. Wilson, J. Phys. Chem. 61 (1978) 714.

${ }^{9}$ E. Ruckenstein, B. Pulvermacher, AICHE J. 19 (1973) 356.

${ }^{10}$ S. Raynaud, E. Champion, D. Bernche-Assollant, Biomaterials 23 (2002) 1073.

${ }^{11}$ W. Tarutis, Jr., Geochim. Cosmochim. Acta 57 (1993) 1349.

12 D. Dollimore, J. Thermal Anal. 38 (1992) 111.

13 B. P. Boudreau, B. R. Ruddick, Am. J. Sci. 291 (1991) 507.

${ }^{14}$ S. Vyazovkin and C. A. Wight, Thermochim. Acta 340 (1999), 53. 